Article

\title{
Extrinsic Value Orientation and Decreased Sustainability of Shared Resources: The Moderating Role of Situational Characteristics
}

\author{
Geurim Han, Junghwa Kim and Sun W. Park * (D) \\ Department of Psychology, Korea University, Seoul 02841, Korea; inpsyght1879@gmail.com (G.H.); \\ junghwakim00@gmail.com (J.K.) \\ * Correspondence: sunwpark@korea.ac.kr; Tel.: +82-2-3290-2868
}

Received: 6 June 2018; Accepted: 25 June 2018; Published: 27 June 2018

check for updates

\begin{abstract}
In the present research, we investigated the roles of both personal values and situational characteristics in predicting environmentally irresponsible behavior using the tragedy of commons dilemma. Graduate or undergraduate students $(n=138)$ visited the laboratory as a group of four and completed measures of personal values and played a commons dilemma game online. Participants were led to believe that they were playing with the other three participants, but they were in fact playing with three bot players that were manipulated to use the shared resources either cooperatively or competitively. It was found that people who put more emphasis on extrinsic values (financial success, appealing appearance, and social recognition) relative to intrinsic values (personal growth, affiliation, and community contribution) consumed more shared resources than their counterparts. However, this link was significant only in the competitive condition, suggesting an interaction between personal and situational factors. Supplementary analyses indicate that financial success and community contribution are particularly important in predicting environmentally irresponsible behavior.
\end{abstract}

Keywords: extrinsic values; intrinsic values; environmentally responsible behavior; tragedy of commons dilemma

\section{Introduction}

The natural environment today is faced with serious threats, such as climate change, melting glaciers, the destruction of the rainforests, and the chemical waste in the oceans, to name a few. With an increased emphasis on environmental protection, the role of psychology has become crucial, as human consumption of natural resources is the primary cause of environmental problems $[1,2]$. In the present research, we investigated the roles of both personal and situational factors in predicting environmentally irresponsible behavior in the context of the tragedy of commons dilemma [3].

\subsection{Environmental Problems as the Tragedy of the Commons}

Many environmental problems in the real world can be understood as a huge commons dilemma in which personal and collective interests are constantly conflicted [4-11]. In the tragedy of the commons dilemma [3], people share limited environmental resources (e.g., trees of the forest) and decide how much of the shared resources they will consume (e.g., felling trees). If individuals pursue their own interests, the shared resources will be quickly depleted and soon there will be no resources left for anyone; in contrast, if they focus on preservation of the shared resources and restrain consumption, they will not make any profit for themselves.

This general property of the commons dilemma can be applied to most environmental problems. Probably due to this similarity, numerous studies $[4,6,7,12-22]$ have used the commons dilemma 
to measure environmentally (ir)responsible behaviors. One finding that many studies consistently observed was that people's environmental behaviors are greatly affected by how others behave. Specifically, when others' behavior was experimentally manipulated to be competitive and take more than their fair share of the shared resources (hereafter called competitive condition), participants also consumed a large portion of the resources; in contrast, when others behaved in a cooperative fashion and reduced consumption of the shared resources (hereafter called cooperative condition), participants' own consumption was restrained as well, such that the resources remained sustainable $[7,14,16,21]$.

To summarize, under competitive situations in which the shared resources are quickly depleted, people in general tend to act according to their own self-interests, adversely affecting the sustainability of the environment. However, it should be noted that previous research has identified several individual difference variables that are associated with resource use in the tragedy of the commons, which are reviewed in the next section.

\subsection{Individual Difference Variables in the Tragedy of the Commons}

A body of studies has investigated the role of personality in the resource use behaviors in the commons dilemma. In one study [12], participants completed a measure of narcissism, formed a group with other participants, and played the tragedy of the commons game until the shared forest became completely depleted or up to 25 rounds. Narcissism was positively related to the amount of harvest in the first round and negatively related to the number of rounds; that is, narcissistic individuals used the shared resources in a selfish fashion, which led to early destruction of the resources. In another study [14], participants completed a measure of the Big Five personality traits and were randomly assigned to a competitive or cooperative condition. In the competitive condition, agreeableness was negatively, and extraversion was positively, related to selfish resource use. No significant results were observed in the cooperative condition.

Although various individual differences were examined in the context of the social dilemmas, values that people consider important in their lives have received particular attention. Social value orientation (SVO) - the tendency to consider others' welfare when allocating resources [23] — is probably the most studied, among others. According to a meta-analysis that included 82 studies [24], SVO was positively related to cooperative behaviors in different types of social dilemmas $(r=0.30)$, and this relation remained significant when only the commons dilemma was taken into account $(r=0.22)$. Other researchers assessed self-transcendence values, including universalism [25], and found that individuals who emphasized transcending their own interests for others showed cooperative resource use such that the shared resources could be sustainably maintained [15,22,26,27].

We want to note that this link between values and resource use in the commons dilemma disappeared when a cooperative context was experimentally induced; that is, when participants were led to believe that others restrained their resource use at the sustainable level, they also restrained their own resource use. Individual differences in values were manifested only when the shared resources were quickly depleted. In one study [7], individuals who were high in SVO consumed the resources with greater self-restraint than those low in SVO when they were assigned to the competitive condition. However, the behavior of the two groups were highly similar in the cooperative condition. Similar results were observed in a more recent study [21]. Pro-environmental values were negatively related to greedy resource use in the competitive condition, but unrelated in the cooperative condition.

When individuals observe others behaving in an environmentally responsible way such that the shared resources are well maintained, there is no conflict between self- and collective interests. However, in a high-threat situation, where the shared resources are constantly overconsumed and their extinction is easily predicted, people start to weigh between self- and collective interests [14]. As a result, values manifest themselves in a way that people who care about the environment reduce resource use, whereas self-interested individuals increase it. In the present study, we investigated how intrinsic and extrinsic values $[28,29]$ interacted with situational characteristic (competitive vs. cooperative) in predicting resource use in the commons dilemma. 


\subsection{Extrinsic Value Orientation and Environmentally Irresponsible Behavior}

Kasser and Ryan [28,29], based on the self-determination theory [30], differentiated extrinsic values from intrinsic ones. Intrinsic values are theorized to be in line with self-actualizing and growth tendencies that are natural to humans, and include personal growth, affiliation, and community contribution. Life with emphasis on those values will likely lead to optimal functioning and well-being on its own (for more detailed discussion, see [31]). In contrast, endorsing or even achieving extrinsic values, such as financial success, appealing appearance, and social recognition, will not be satisfying in itself because they require approval or attention from others. Thus, extrinsic values are essentially based on intense self-interest, competition with others, and the desire for success [32-34].

Previous research has indeed found that extrinsic value orientation (EVO)—relative importance of extrinsic values to intrinsic values-was negatively related to the willingness to pay to protect the environment and self-rated environmentally responsible behavior [32,34]. In a 2-year longitudinal study [35], EVO, environmental worldviews, and environmental identification were included in a structural equation model to predict self-rated environmentally responsible behavior. Two years later, EVO prospectively predicted environmentally responsible behavior, but environmental worldviews and environmental identification did not, indicating the crucial role of EVO in predicting environmentally responsible behavior.

Moreover, in another study [20], EVO predicted actual, not self-rated, resource use behavior in the commons dilemma. In Study 1, participants imagined that they played the commons dilemma game with three other participants. The amount of the first round's harvest was positively related to EVO. When EVO and SVO were controlled for each other, only EVO remained as a significant predictor. Participants in Study 2 were told to play the commons dilemma game with three other random participants, but were in fact assigned to either a high (four participants high in EVO), low (four low in EVO), or mixed (two high and two low in EVO) EVO group. The dilemma game continued until the shared resources became completely depleted or up to 25 rounds. At the individual level, EVO was once again positively related to the first-round harvest. At the group level, however, the total amount of harvest was lowest in the group with four participants high in EVO because their shared resources were depleted quicker than those of the other two groups. Thus, in the long-term, resource use behavior of individuals high in EVO is destructive not only to the environment but also to themselves. Taken together, the results from the previous studies suggest that individual differences in EVO can be quite a powerful predictor of environmentally irresponsible behavior.

\subsection{The Present Research}

The present research aimed to examine the interaction effect between personal values (EVO) and situational characteristic (competitive vs. cooperative) on resource use behavior in the commons dilemma. Participants came to a lab as a group of four, completed a measure of personal values, and played a real-time online program FISH 5.0 [36] that simulated the commons dilemma. Although participants were led to believe that they were playing with the other three participants, they were in fact playing with three bot players that were manipulated to behave either in a competitive or cooperative fashion. We hypothesized that EVO would be positively related to greedy (i.e., environmentally irresponsible) resource use in the competitive condition, but unrelated in the cooperative condition. In addition, given that previous studies using EVO did not report results regarding individual values that constitute EVO, except for Unanue et al. [35], we performed supplementary analyses using the individual values.

\section{Method and Materials}

\subsection{Participants and Procedure}

The Institutional Review Board at Korea University reviewed this study protocol, and approval was obtained. Following the recommendations of Simmons, Nelson, and Simonsohn [37], we report 
how we determined our sample size, all data exclusions, all manipulations, and all measures in the study. A power analysis using $G^{*}$ Power [38] indicated that we needed 88 participants to detect the effect size reported in Sussman et al. [21] ( $d=0.6,80 \%$ power, $\alpha=0.05$, two-tailed). We predetermined the recruitment of at least 88 participants, but aimed for as many as possible within three weeks. Online advertisements were posted on a community website at a private university in South Korea. A total of 138 graduate or undergraduate students were recruited ( 57 males, 81 females), and their mean age was $23.01(S D=2.24)$. However, data from two participants in FISH 5.0 were not saved due to unknown technical error, so those data were dropped in the analyses. All the participants were Korean, and materials were written in Korean.

Four participants were invited for each experimental session. As soon as they arrived in the lab, they were seated in a partitioned cubicle so that they could not see the faces of others. They were told that the purpose of the study was to investigate the effect of personality on decision making, and they would complete a battery of questionnaires and play a decision-making game (a commons dilemma game using FISH 5.0) with the other three participants, but they were in fact playing with virtual bot players programmed by the experimenter. In each session, two of the four participants were assigned to a cooperative condition and the other two a competitive condition (see below for detailed information). When a session did not have four participants, up to three confederates joined to eliminate any suspicions that they might play with computerized players.

All procedures were performed on computers in a cubicle. In the first part of the session, participants completed a packet of questionnaires including a measure of intrinsic and extrinsic values, along with other personality questionnaires to disguise the purpose of the study. Next, they watched a 5-min FISH 5.0 game tutorial video clip created by us to learn how to play the game and were informed the goal of the game was to get as much profit as possible while keeping resources from getting depleted. After watching the clip, the experimenter made sure that all participants fully understood the rules of the game and answered any questions that they had. Participants had to complete 10 seasons to finish the game, but they were not told in advance when the game would end. After all participants finished the game, they were debriefed, thanked, and compensated for their participation (7000 KRW, approximately 7 USD).

Care was taken to make participants believe that they were playing with the other three participants. First, we learned that the discrepancy between bot players' fishing action and mouse-clicking sound raised suspicion during pilot trials, so we replaced the computer mice with silent ones. Second, the experimenter repeatedly warned that they should start on her call. Finally, when participants finished their game, a screen popped up to tell them that it would take some time for the data to be safely saved, which was in fact an excuse for time lag between participants.

\subsection{Materials}

\subsubsection{Extrinsic Value Orientation}

A Korean version of the 47-item Aspiration Index [39] was used to assess values. Although this index measures 11 different values, only 3 intrinsic (personal growth, affiliation, and community contribution) and 3 extrinsic (financial success, appealing appearance, and social recognition) values were included in our analyses, as in previous studies [20,32,34,35]. Participants rated the importance of each value on a scale from 1 (totally unimportant) to 7 (extremely important). Example items are "To overcome challenges that life presents me" (personal growth), "To feel that there are people who really love me" (affiliation), "To help the world become a better place" (community contribution), "To have many expensive possessions" (financial success), "To successfully hide the signs of aging" (appealing appearance), and "To be admired by many people" (social recognition).

Researchers on values argue for use of the relative, not absolute, importance of values. Different values can affect attitudes or behaviors simultaneously, so what is critical is the strength of one value compared to others $[25,28,29,40]$. In the present study, EVO-relative importance of extrinsic 
to intrinsic values-was computed by subtracting mean scores of three intrinsic values from the mean scores of extrinsic values following Sheldon and McGregor [20]. The resulting positive scores indicate more emphasis on extrinsic than intrinsic values, whereas negative scores indicate more emphasis on intrinsic than extrinsic values.

As mentioned earlier, we conducted supplementary analyses to examine the role of each of the six values on greediness in addition to EVO. For these analyses, we used mean-corrected scores to represent the relative importance [39]. Specifically, the grand mean of all 47 items was subtracted from the mean score of each value.

\subsubsection{FISH 5.0 and Greediness}

FISH 5.0 is an online program developed to conduct studies of commons dilemmas by Gifford and Aranda [36]. In this program, four fishers share an ocean that can accommodate up to 80 fish and make their living by catching fish. During each fishing session, they can go out to the sea and catch as many fish as they want. One particular strength of this program is that participants can see how many fish the four players have caught and how many are left in the ocean in real time. With the assistance of the developers, we translated the program into Korean (for detailed description of the program, see [36]).

After a fishing session, and before the next one, the number of fish left in the ocean is doubled, but only up to 80 fish. Thus, the maximum number of fish they can catch per season to maintain a sustainable ocean is 40 (10 fish per fisher). Each fish is worth 200,000 KRW (approximately 200 USD), such that fishers can earn the national average income of Korea if they catch 10 fish per season. Fishers need to decide how many fish they will catch for each season, considering their own interests and the sustainability of the shared ocean.

Greediness was the main dependent variable in the present study, which conceptually refers to the extent to which a fisher pursues his or her own profit rather than the sustainability of the ocean. A greediness score of 0 indicates that a fisher does not catch any fish. A score of 0.5 indicates that a fisher catches fish at an exactly sustainable rate (10 fish when there are 80). A score of 1 indicates that a fisher catches every possible fish given all four fishers take the same number of fish (20 fish). A score higher than 1 indicates that a fisher catches fish more than his or her fair share (more than 20 fish). Both greediness of season 1 and mean greediness of 10 seasons were used for analyses [12,20,21].

Participants were told that they would play with the other three participants, but in fact each one played with three bot players. How many fish those bot players catch (i.e., greediness) was programmed to maintain the ocean either to be sustainable or unsustainable, following Sussman and colleagues [21].

In addition, FISH 5.0 has several different functions that control bot players' fishing behavior (e.g., how quickly they catch fish). All these functions were carefully manipulated through many test trials to produce human-like bot actions, which was aimed to make participants believe that they are playing with other participants, not artificial players.

\section{Results}

\subsection{Preliminary Analyses}

Descriptive statistics and correlations between the study variables are presented in Table 1. We conducted a series of multiple regressions to test three-way $(\mathrm{EVO} \times$ condition $\times$ participant gender) interactions, with greediness as a dependent variable. None of the interaction terms including gender were significant, so all the analyses reported below were collapsed across gender. 
Table 1. Descriptive statistics and correlations between variables.

\begin{tabular}{lcccccccccc}
\hline \multicolumn{1}{c}{ Variable } & $\mathbf{1}$ & $\mathbf{2}$ & $\mathbf{3}$ & $\mathbf{4}$ & $\mathbf{5}$ & $\mathbf{6}$ & $\mathbf{7}$ & $\mathbf{8}$ & $\mathbf{9}$ & $\boldsymbol{M}(\mathrm{SD})$ \\
\hline 1. EVO & - & $0.72^{* * *}$ & $0.81^{* * *}$ & $0.40^{* * *}$ & $-0.36^{* *}$ & $-0.46^{* * *}$ & $-0.63^{* * *}$ & -0.02 & -0.01 & $-0.70(0.87)$ \\
2. Financial success & $0.2^{* * *}$ & - & $0.48^{* * *}$ & 0.12 & 0.06 & $-0.41^{* * *}$ & $-0.28^{*}$ & -0.02 & 0.07 & $0.03(0.97)$ \\
3. Appearance & $0.67^{* * *}$ & $0.44^{* * *}$ & - & 0.18 & $-0.33^{* *}$ & $-0.30^{* *}$ & $-0.48^{* * *}$ & -0.11 & -0.02 & $-0.55(0.72)$ \\
4. Social recognition & $0.54^{* * *}$ & $0.35^{* *}$ & 0.12 & - & $-0.24^{*}$ & -0.03 & 0.01 & 0.01 & 0.03 & $-0.02(0.68)$ \\
5. Personal growth & $-0.51^{* * *}$ & $-0.35^{* *}$ & $-0.33^{* *}$ & -0.17 & - & -0.09 & 0.18 & 0.04 & 0.05 & $0.59(0.48)$ \\
6. Affiliation & $-0.54^{* * *}$ & $-0.36^{* *}$ & $-0.33^{* *}$ & -0.03 & $0.32^{* *}$ & - & -0.03 & -0.07 & 0.01 & $0.86(0.64)$ \\
7. Community & $-0.78^{* * *}$ & $-0.57^{* *}$ & $-0.46^{* * *}$ & $-0.30^{*}$ & 0.17 & $0.29^{*}$ & - & -0.02 & 0.08 & $0.09(0.97)$ \\
8. Greediness of season 1 & $0.38^{* *}$ & $0.33^{* *}$ & 0.12 & 0.19 & $-0.27^{*}$ & -0.20 & $-0.35^{* *}$ & - & 0.17 & $0.66(0.31)$ \\
9. Greediness mean & $0.27^{*}$ & $0.27^{*}$ & 0.05 & 0.19 & -0.17 & -0.14 & -0.20 & $0.73^{* * *}$ & - & $0.59(0.20)$ \\
\hline$M$ & -0.60 & 0.13 & -0.48 & -0.01 & 0.55 & 0.84 & 0.04 & 0.72 & 0.68 & - \\
$S D$ & 1.02 & 0.94 & 0.74 & 0.85 & 0.46 & 0.62 & 0.98 & 0.40 & 0.38 \\
\hline
\end{tabular}

Note: $\mathrm{EVO}=$ extrinsic value orientation. Results for the cooperative condition are presented above the diagonal and results for the competitive condition are presented below the diagonal. ${ }^{*} p \leq 0.05,{ }^{* *} p \leq 0.01,{ }^{* * *} p \leq 0.001$.

The number of fish available in the ocean at the start of each session is depicted in Figure 1, where high and low EVO values are indexed at $1 S D$ above and below the mean. The two lines at the top, observed in the cooperative condition, show that the sustainability of the ocean was maintained consistently at a high level, and 68 fish remained after the final 10th season. In contrast, the two lines at the bottom, observed in the competitive condition, indicates that the average number of fish was depleted very quickly as the season progressed, resulting in only 19 fish left in the end. More importantly, the discrepancy between high and low EVO stood out only in the competitive condition, raising the possibility that individual differences in EVO could interact with situational differences, which we tested below.

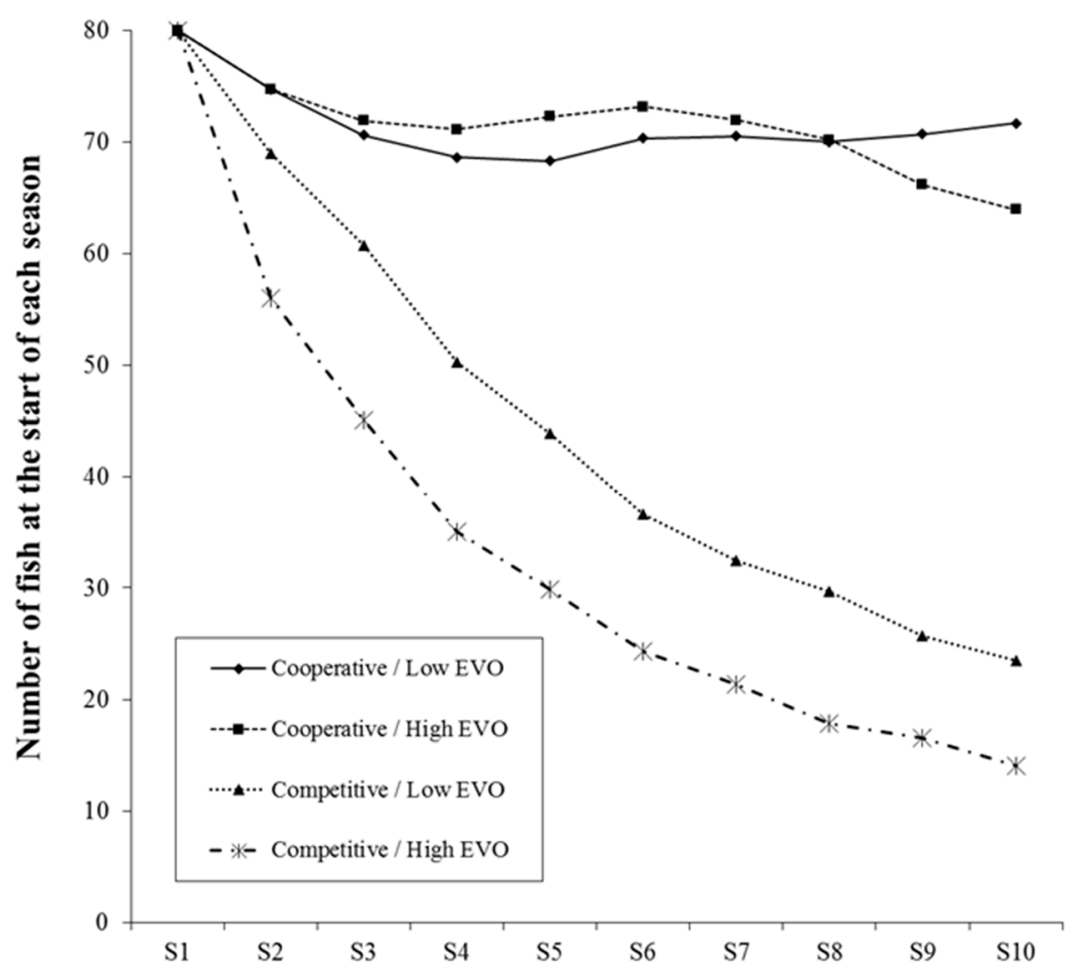

Figure 1. Average number of fish available in the ocean at the start of each season by condition and EVO. High and low EVO values are indexed at $1 S D$ above and below the mean. EVO = extrinsic value orientation.

\subsection{Primary Analyses}

The main question of the present study is whether the relation between EVO and greediness differs depending on the characteristics of situations (cooperative vs. competitive). To answer the question, 
we conducted moderation analyses using the SPSS macro PROCESS (Model 1; [41]). Specifically, we put $\mathrm{EVO}$ as the independent variable and condition (cooperative $=0$; competitive $=1$ ) as the moderator to predict greediness.

As shown in Table 2, when the dependent variable was greediness of season 1, the interaction between EVO and condition was significant. Simple slope tests were performed at $1 S D$ above and below the mean to further understand the nature of this interaction (Figure 2). As expected, EVO was positively related to greediness in the competitive condition, but unrelated in the cooperative condition. With mean greediness as the dependent variable (Table 3), the interaction was not significant $(p=0.06)$. Still, we further performed simple slope analyses at $1 S D$ above and below the mean to see whether simple slopes were significant in the expected direction (Figure 3). Once again, the positive link between EVO and greediness was significant only in the competitive condition. These results indicate that people high in EVO consumed more resources than those low in EVO only when they were in the competitive condition, supporting our main hypothesis.

Table 2. Interaction and simple slope analyses predicting greediness of season 1.

\begin{tabular}{|c|c|c|c|c|c|c|c|c|c|}
\hline \multirow{3}{*}{ Variable } & \multicolumn{3}{|c|}{ Interaction } & \multicolumn{6}{|c|}{ Simple Slope } \\
\hline & \multirow{2}{*}{$b(S E)$} & \multirow{2}{*}{$p$} & \multirow{2}{*}[95\%\mathrm{CI}]{} & \multicolumn{3}{|c|}{ Cooperative Condition } & \multicolumn{3}{|c|}{ Competitive Condition } \\
\hline & & & & $b(S E)$ & $p$ & {$[95 \% \mathrm{CI}]$} & $b(S E)$ & $p$ & {$[95 \% \mathrm{CI}]$} \\
\hline EVO & $0.15(0.06)$ & 0.02 & {$[0.03,0.28]$} & $-0.01(0.05)$ & 0.91 & {$[-0.10,0.09]$} & $0.15(0.04)$ & $<0.01$ & {$[0.06,0.23]$} \\
\hline \multicolumn{10}{|l|}{ Extrinsic value } \\
\hline Financial success & $0.14(0.06)$ & 0.02 & {$[0.02,0.27]$} & $-0.01(0.04)$ & 0.87 & {$[-0.09,0.08]$} & $0.14(0.04)$ & $<0.01$ & {$[0.05,0.23]$} \\
\hline Appearance & $0.11(0.08)$ & 0.20 & {$[-0.06,0.28]$} & $-0.05(0.06)$ & 0.45 & {$[-0.17,0.07]$} & $0.06(0.06)$ & 0.29 & {$[-0.05,0.18]$} \\
\hline Social recognition & $0.08(0.08)$ & 0.32 & {$[-0.08,0.24]$} & $0.01(0.06)$ & 0.92 & {$[-0.12,0.13]$} & $0.09(0.05)$ & 0.09 & {$[-0.01,0.19]$} \\
\hline \multicolumn{10}{|l|}{ Intrinsic value } \\
\hline Personal growth & $-0.26(0.13)$ & 0.05 & {$[-0.52,-0.01]$} & $0.02(0.09)$ & 0.79 & {$[-0.15,0.20]$} & $-0.24(0.09)$ & 0.01 & {$[-0.42,-0.05]$} \\
\hline Affiliation & $-0.09(0.10)$ & 0.34 & {$[-0.29,0.10]$} & $-0.03(0.07)$ & 0.63 & {$[-0.17,0.10]$} & $-0.13(0.07)$ & 0.07 & {$[-0.26,0.01]$} \\
\hline Community & $-0.13(0.06)$ & 0.03 & {$[-0.25,-0.01]$} & $-0.01(0.04)$ & 0.87 & {$[-0.09,0.08]$} & $-0.14(0.04)$ & $<0.01$ & {$[-0.23,-0.06]$} \\
\hline
\end{tabular}

Note: $\mathrm{EVO}=$ extrinsic value orientation. Unstandardized coefficients are reported; $\mathrm{CI}$ = confidence interval.

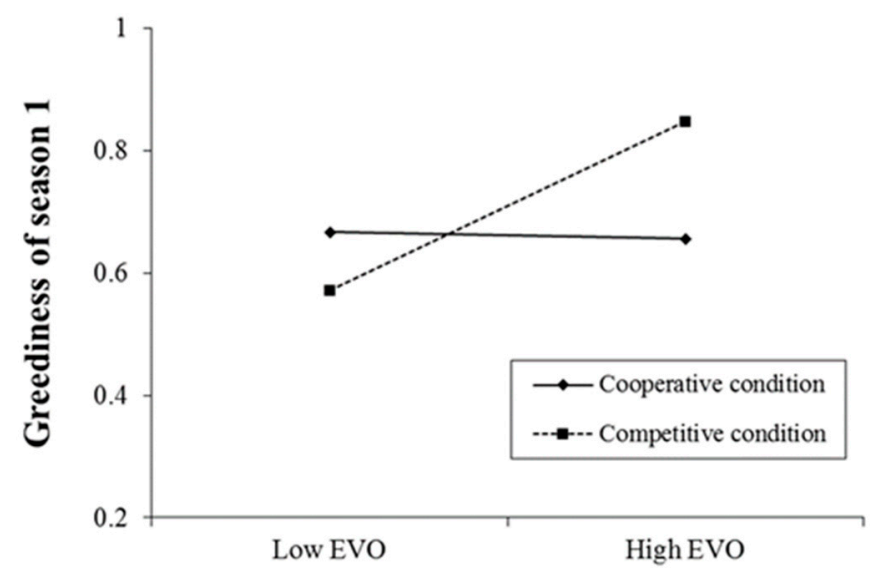

Figure 2. Greediness of season 1 as a function of condition and EVO. High and low EVO values are indexed at $1 S D$ above and below the mean. EVO = Extrinsic value orientation. 
Table 3. Interaction and simple slope analyses predicting mean greediness.

\begin{tabular}{|c|c|c|c|c|c|c|c|c|c|}
\hline \multirow{3}{*}{ Variable } & \multicolumn{3}{|c|}{ Interaction } & \multicolumn{6}{|c|}{ Simple Slope } \\
\hline & \multirow{2}{*}{$b(S E)$} & \multirow{2}{*}{$p$} & \multirow{2}{*}[95\%\mathrm{CI}]{} & \multicolumn{3}{|c|}{ Cooperative Condition } & \multicolumn{3}{|c|}{ Competitive Condition } \\
\hline & & & & $b(S E)$ & $p$ & {$[95 \% \mathrm{CI}]$} & $b(S E)$ & $p$ & {$[95 \% \mathrm{CI}]$} \\
\hline EVO & $0.10(0.05)$ & 0.06 & {$[-0.01,0.21]$} & $0.00(0.04)$ & 0.95 & {$[-0.08,0.08]$} & $0.10(0.04)$ & $<0.01$ & {$[0.03,0.17]$} \\
\hline \multicolumn{10}{|l|}{ Extrinsic value } \\
\hline Financial success & $0.10(0.05)$ & 0.07 & {$[-0.01,0.20]$} & $0.01(0.04)$ & 0.72 & {$[-0.06,0.09]$} & $0.11(0.04)$ & $<0.01$ & {$[0.03,0.18]$} \\
\hline Appearance & $0.03(0.07)$ & 0.69 & {$[-0.11,0.17]$} & $0.00(0.05)$ & 0.93 & {$[-0.11,0.10]$} & $0.02(0.05)$ & 0.64 & {$[-0.07,0.12]$} \\
\hline Social recognition & $0.08(0.07)$ & 0.28 & {$[-0.06,0.21]$} & $0.01(0.05)$ & 0.87 & {$[-0.10,0.11]$} & $0.08(0.04)$ & 0.05 & {$[0.00,0.17]$} \\
\hline \multicolumn{10}{|l|}{ Intrinsic value } \\
\hline Personal growth & $-0.16(0.11)$ & 0.16 & {$[-0.38,0.06]$} & $0.02(0.08)$ & 0.79 & {$[-0.13,0.17]$} & $-0.14(0.08)$ & 0.09 & {$[-0.30,0.02]$} \\
\hline Affiliation & $-0.09(0.08)$ & 0.28 & {$[-0.25,0.07]$} & $0.00(0.06)$ & 0.95 & {$[-0.11,0.12]$} & $-0.09(0.06)$ & 0.14 & {$[-0.20,0.03]$} \\
\hline Community & $-0.09(0.05)$ & 0.08 & {$[-0.20,0.01]$} & $0.02(0.04)$ & 0.69 & {$[-0.06,0.09]$} & $-0.08(0.04)$ & 0.04 & {$[-0.15,-0.01]$} \\
\hline
\end{tabular}

Note: $\mathrm{EVO}=$ extrinsic value orientation. Unstandardized coefficients are reported; $\mathrm{CI}=$ confidence interval.

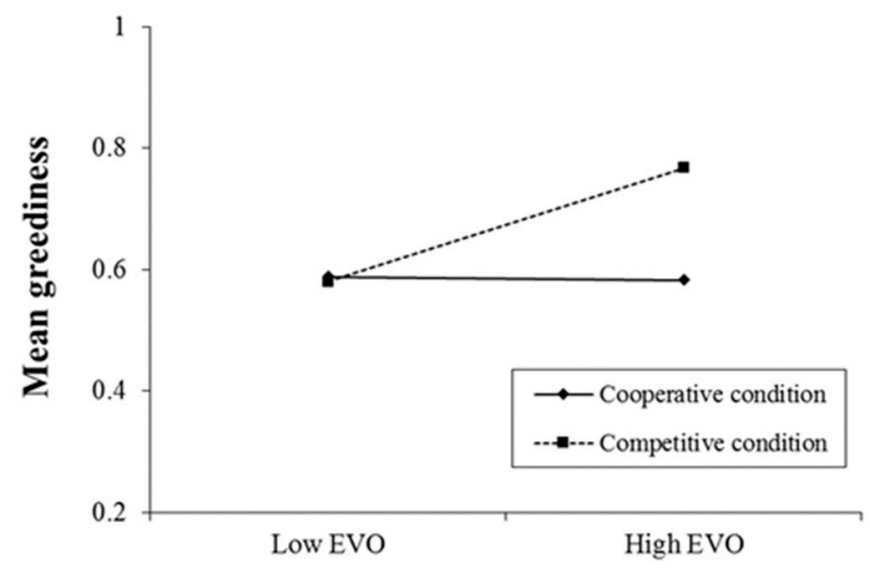

Figure 3. Mean greediness as a function of condition and EVO. High and low EVO values are indexed at $1 S D$ above and below the mean. EVO = Extrinsic value orientation.

\subsection{Supplementary Analyses}

Next, we conducted supplementary analyses to examine the effects of each value on fishing behavior. We put six values (three intrinsic and three extrinsic), one at a time, as the independent variable, and the condition as the moderator. We conducted simple slope analyses regardless of whether the interaction was significant, as our main interests resided in the competitive condition.

The results of the interaction and simple slope analyses with greediness of season 1 as the dependent variable are presented in Table 2. In the competitive condition, financial success was positively, and personal growth and community contribution were negatively, related to greediness. No significant results were observed in the cooperative condition. Table 3 presents the results of the interaction and simple slope analyses when mean greediness was the dependent variable. Financial success and social recognition were positively, and community contribution was negatively, related to greediness in the competitive condition. No values were significantly related to greediness in the cooperative condition.

Taken together, these results indicate that people who put great importance in extrinsic values, particularly financial success and social recognition, tended to consume more shared resources when they realized that others were not cooperative in conserving the resources. In contrast, people who emphasized intrinsic values, such as personal growth and community contribution, restrained their consumption, even when others were greedily consuming the shared resources. 


\section{Discussion}

With serious environmental problems around the world, it is becoming more and more important to preserve natural resources and encourage environmentally responsible behavior. In the present study, we investigated both personal and situational factors that are associated with environmentally irresponsible behavior and thereby decreased sustainability of public resources. Specifically, using the tragedy of commons dilemma [3] that mirrors real-life environmental problems, we developed two different situations: the one where others behave cooperatively such that the shared resources are sustainably maintained, and the other where others behave competitively such that the shared resources are quickly depleted. As for the individual difference variables, EVO was assessed, given many previous studies documenting its detrimental effects on the environment $[20,32,34,35]$.

\subsection{Key Findings and Contributions}

The positive link between EVO and greedy resource use was observed when others behaved competitively, but not when others were cooperative in preserving the shared resources, regardless of whether greediness was assessed as the greediness of season 1 or mean greediness across 10 seasons. This finding is consistent with previous studies $[7,14,21]$ and was also observed when individual values were examined. For individual values, financial success was positively, and community contribution was negatively, related to both types of greediness. In addition, personal growth was negatively related to the greediness of season 1 , and social recognition was positively related to the mean for greediness. Taken together, these results indicate that individuals who care about extrinsic values more than intrinsic values tend to consume more shared resources, particularly when the resource is quickly depleted.

Individuals who pursue extrinsic values that do not inherently fulfill basic psychological needs suffer from insecurity and instability, so they need external approval or attention to maintain their self-worth $[30,31]$. When the shared resources are depleted, extrinsically oriented individuals presumably felt a threat to their self-worth and therefore greatly consumed the shared resources to protect it, even if it led to complete exhaustion of the resources. In contrast, those who pursue intrinsic values were not affected by the situation. As shown in Figures 2 and 3, their resource use remained relatively low, even when others were greedily consuming the shared resources, because their self-worth resides inside of themselves.

We investigated EVO among many individual difference variables. Previous studies have found that EVO has better predictive validity than SVO, environmental worldviews, or environmental identification $[20,35]$. Although we did not include those related variables in our study, our results also attested to the important role of EVO in environmental protection. Thus, researchers who want to intervene to protect the environment may want to focus on EVO (for a detailed discussion, see [42]). Importantly, under contemporary capitalism, the mainstream media constantly send out messages that wealth is the indicator of a successful life and associate happiness with luxury goods and appealing appearance, which unfortunately leads to both psychological and environmental malfunctioning $[28,29,33]$. Researchers or social activists need to develop a way, despite the pervasiveness of capitalism, to switch people's focus from extrinsic to intrinsic values.

Although previous studies have demonstrated that EVO is a strong predictor of environmentally irresponsible behavior, they did not report how each of individual values that constitute EVO is related to it $[20,32,34]$. Thus, it was not clear whether the effects of EVO come from intrinsic values, extrinsic values, or a combination of the two. In an exceptional study that conducted analyses using individual values [35], environmentally responsible behavior was positively related to community contribution, but negatively related to financial success. Unexpectedly, it was positively related to social recognition. Combined with our results, it can be tentatively concluded that financial success and community contribution are the two best predictors of environmentally responsible behavior. We encourage other researchers to report these results so that we can better understand the role of values in environmental problems. 
One merit of the present study is the use of greediness, instead of absolute amount, of resource use in the commons dilemma [21]. When the shared resources become depleted, it is only natural that people reduce the absolute amount of consumption because the resources themselves decline. Thus, if researchers compare the absolute numbers of fish caught in competitive and cooperative conditions, particularly in the later rounds, they must wrongly conclude that resource use is less problematic in the competitive than in the cooperative condition. For example, imagine there are 80 fish in the ocean and the number of fish remaining in the ocean is doubled in the next season, just like in FISH 5.0. In this case, catching 30 fish can be considered environmentally responsible because the ocean can contain 80 fish, the maximum number, next season. However, catching 20 fish when there are 30 fish left in the ocean can be irresponsible because it will make the shared resources unsustainable. Greediness in the present study was calculated relative to the number of fish available in the ocean per season [36]. We believe that greediness assessed this way better represents environmentally irresponsible behavior than the absolute amount.

Finally, participants in the present study were Korean. Although there is some evidence that young Koreans are becoming individualistic [43,44], Koreans have long been considered collectivists who put collective interests ahead of personal ones [45]. Still, we found that personal values played an important role in predicting environmentally responsible behavior. This finding suggests that the links between personal values and environmental behaviors are more universal than culture-specific (for discussion on universality of values see [39]).

\subsection{Limitations and Suggestions for Future Research}

Although the present research provided important insights, several limitations need to be mentioned. First, we did not empirically examine the underlying psychological mechanism of the link between EVO and environmentally irresponsible behavior, and some readers may offer alternative explanations. Specifically, the positive association between financial success and environmentally irresponsible behavior (i.e., greediness) observed in the present study may have been simply due to the characteristic of the commons dilemma that catching more fish meant earning more money. Thus, in a different task where, for example, participants are asked to save as much money as possible for a week, financial success may be negatively related to environmentally irresponsible behavior; in other words, those who care about financial success may display energy-efficient behaviors. However, this explanation cannot explain why financial success was unrelated to greediness in the cooperative condition where more fish were available. Based on the self-determination theory [30], we speculate that those high in EVO felt a threat to their self-worth when the resources were quickly depleted, and they were motivated to take as much as possible. Future research should directly examine the underlying mechanism by analyzing, for example, verbal or physiological responses during the dilemma game.

Also, readers may wonder whether our results can be accounted for by conformity to others [16]. However, this alternative idea can explain only half of the findings. Specifically, conformity can explain why participants in general were greedier in the competitive than in the cooperative condition, as the bot players (i.e., others in the mind of participants) were greedier in the competitive condition. However, conformity cannot explain why those high in EVO were greedier than those low in EVO or the bot players. As mentioned earlier, this finding can be explained by them being motivated to pursue their own self-interests, even at the cost of losing the shared resources.

External validity and generalizability of the present findings should be addressed as well. Although the commons dilemma conceptually represents the real-world environmental problems and is a vastly used paradigm $[4,6,7,12-22]$, there is no guarantee that greedy behaviors observed in the dilemma are actually manifested outside a psychology lab. Also, only young adults were recruited in the present study. As previous research has found that older people tend to show less interests in extrinsic values than younger ones [46,47], future researchers are encouraged to utilize more realistic behavioral measures with a wider range of age groups. 
Finally, we are cautious about making a conclusion that EVO is a causal factor of environmental problems or environmental irresponsible behaviors solely based on the present research. To ensure the direction of causality, further studies involving manipulation of extrinsic vs. intrinsic values (e.g., value affirmation) may be needed. These attempts will be also helpful in understanding how and where to intervene to increase environmental sustainability.

Author Contributions: All three authors worked together to develop the study design; Geurim Han and Junghwa Kim collected and analyzed the data; Geurim Han and Sun W. Park collaborated on writing the article; Junghwa Kim reviewed it.

Funding: This study was supported by the Ministry of Education of the Republic of Korea and the National Research Foundation of Korea (NRF-2017S1A5A2A03068974), and OJeong Eco-Resilience Institute at Korea University.

Conflicts of Interest: The authors declare no conflict of interest.

\section{References}

1. Clayton, S.; Brook, A. Can psychology help save the world? A model for conservation psychology. Anal. Soc. Issues Public Policy 2005, 5, 87-102. [CrossRef]

2. Saunders, C.D. The emerging field of conservation psychology. Hum. Ecol. Rev. 2003, 10, 137-149.

3. Hardin, G. The tragedy of the commons. Science 1968, 162, 1243-1244. [CrossRef] [PubMed]

4. Brewer, M.B.; Kramer, R.M. Choice behavior in social dilemmas: Effects of social identity, group size, and decision framing. J. Pers. Soc. Psychol. 1986, 50, 543-549. [CrossRef]

5. Hine, D.W.; Gifford, R. Individual restraint and group efficiency in commons dilemmas: The effects of two types of environmental uncertainty. J. Appl. Soc. Psychol. 1996, 26, 993-1009. [CrossRef]

6. Jager, W.; Janssen, M.A.; Vlek, C.A. How uncertainty stimulates over-harvesting in a resource dilemma: Three process explanations. J. Environ. Psychol. 2002, 22, 247-263. [CrossRef]

7. Kramer, R.M.; McClintock, C.G.; Messick, D.M. Social values and cooperative response to a simulated resource conservation crisis. J. Pers. 1986, 54, 576-582. [CrossRef]

8. Kortenkamp, K.V.; Moore, C.F. Time, uncertainty, and individual differences in decisions to cooperate in resource dilemmas. Pers. Soc. Psychol. Bull. 2006, 32, 603-615. [CrossRef] [PubMed]

9. O'Connor, B.P.; Tindall, D.B. Attributions and behavior in a commons dilemma. J. Psychol. 1990, 12, 485-494. [CrossRef]

10. Smith, J.M.; Bell, P.A. Environmental concern and cooperative-competitive behavior in a simulated commons dilemma. J. Soc. Psychol. 1992, 132, 461-468. [CrossRef] [PubMed]

11. Van Vugt, M. Averting the tragedy of the commons: Using social psychological science to protect the environment. Curr. Dir. Psychol. Sci. 2009, 18, 169-173. [CrossRef]

12. Campbell, W.K.; Bush, C.P.; Brunell, A.B.; Shelton, J. Understanding the social costs of narcissism: The case of the tragedy of the commons. Pers. Soc. Psychol. Bull. 2005, 31, 1358-1368. [CrossRef] [PubMed]

13. Kaiser, F.G.; Byrka, K. Environmentalism as a trait: Gauging people's prosocial personality in terms of environmental engagement. Int. J. Psychol. 2011, 46, 71-79. [CrossRef] [PubMed]

14. Koole, S.L.; Jager, W.; van den Berg, A.E.; Vlek, C.A.; Hofstee, W.K. On the social nature of personality: Effects of extraversion, agreeableness, and feedback about collective resource use on cooperation in a resource dilemma. Pers. Soc. Psychol. Bull. 2001, 27, 289-301. [CrossRef]

15. Parks, C.D. The predictive ability of social values in resource dilemmas and public goods games. Pers. Soc. Psychol. Bull. 1994, 20, 431-438. [CrossRef]

16. Rutte, C.G.; Wilke, H.A.; Messick, D.M. Scarcity or abundance caused by people or the environment as determinants of behavior in the resource dilemma. J. Exp. Soc. Psychol. 1987, 23, 208-216. [CrossRef]

17. Samuelson, C.D.; Messick, D.M. Alternative structural solutions to resource dilemmas. Organ. Behav. Hum. Decis. Process. 1986, 37, 139-155. [CrossRef]

18. Samuelson, C.D.; Messick, D.M. Inequities in access to and use of shared resources in social dilemmas. J. Pers. Soc. Psychol. 1986, 51, 960-967. [CrossRef]

19. Samuelson, C.D.; Messick, D.M.; Rutte, C.; Wilke, H. Individual and structural solutions to resource dilemmas in two cultures. J. Pers. Soc. Psychol. 1984, 47, 94-104. [CrossRef] 
20. Sheldon, K.M.; McGregor, H.A. Extrinsic value orientation and "the tragedy of the commons". J. Pers. 2000, 68, 383-411. [CrossRef] [PubMed]

21. Sussman, R.; Lavallee, L.F.; Gifford, R. Pro-environmental values matter in competitive but not cooperative commons dilemmas. J. Soc. Psychol. 2016, 156, 43-55. [CrossRef] [PubMed]

22. Van Vugt, M.; Meertens, R.M.; Lange, P.A. Car versus public transportation? The role of social value orientations in a real-life social dilemma. J. Appl. Soc. Psychol. 1995, 25, 258-278. [CrossRef]

23. Messick, D.M.; McClintock, C.G. Motivational bases of choice in experimental games. J. Exp. Soc. Psychol. 1968, 4, 1-25. [CrossRef]

24. Balliet, D.; Parks, C.; Joireman, J. Social value orientation and cooperation in social dilemmas: A meta-analysis. Gr. Process. Intergr. Relat. 2009, 12, 533-547. [CrossRef]

25. Schwartz, S.H. Universals in the content and structure of values: Theoretical advances and empirical tests in 20 countries. Adv. Exp. Soc. Psychol. 1992, 25, 1-65. [CrossRef]

26. Gärling, T. Value priorities, social value orientations and cooperation in social dilemmas. Br. J. Soc. Psychol. 1999, 38, 397-408. [CrossRef]

27. Joireman, J.A.; Lasane, T.P.; Bennet, J.; Richards, D.; Solaimani, S. Integrating social value orientation and the consideration of future consequences within the extended norm activation model of proenvironmental behaviour. Br. J. Soc. Psychol. 2001, 40, 133-155. [CrossRef] [PubMed]

28. Kasser, T.; Ryan, R.M. A dark side of the American dream: Correlates of financial success as a central life aspiration. J. Pers. Soc. Psychol. 1993, 65, 410-422. [CrossRef] [PubMed]

29. Kasser, T.; Ryan, R.M. Further examining the American dream: Differential correlates of intrinsic and extrinsic goals. Persoanl. Soc. Psychol. Bull. 1996, 22, 280-287. [CrossRef]

30. Deci, E.L.; Ryan, R.M. The "what" and "why" of goal pursuits: Human needs and the self-determination of behavior. Psychol. Inq. 2000, 11, 227-268. [CrossRef]

31. Ryan, R.M.; Huta, V.; Deci, E. Living well: A self-determination theory perspective on eudaimonia. J. Happiness Stud. 2008, 9, 139-170. [CrossRef]

32. Brown, K.W.; Kasser, T. Are psychological and ecological well-being compatible? The role of values, mindfulness, and lifestyle. Soc. Indic. Res. 2005, 74, 349-368. [CrossRef]

33. Kasser, T.; Cohn, S.; Kanner, A.D.; Ryan, R.M. Some costs of American corporate capitalism: A psychological exploration of value and goal conflicts. Psychol. Inq. 2007, 18, 1-22. [CrossRef]

34. Ku, L.; Zaroff, C. How far is your money from your mouth? The effects of intrinsic relative to extrinsic values on willingness to pay and protect the environment. J. Environ. Psychol. 2014, 40, 472-483. [CrossRef]

35. Unanue, W.; Vignoles, V.L.; Dittmar, H.; Vansteenkiste, M. Life goals predict environmental behavior: Cross-cultural and longitudinal evidence. J. Environ. Psychol. 2016, 46, 10-22. [CrossRef]

36. Gifford, R.; Aranda, J. Fish 4.0 User's Manual. Available online: http://web.uvic.ca/ esplab/sites/default/ files/FISH\%20MANUAL\%20-\%20updated\%20final_0.pdf (accessed on 5 June 2018).

37. Simmons, J.P.; Nelson, L.D.; Simonsohn, U. A 21 Word Solution. Available online: https://ssrn.com/ abstract $=2160588$ (accessed on 5 June 2018).

38. Faul, F.; Erdfelder, E.; Buchner, A.; Lang, A.G. Statistical power analyses using G* Power 3.1: Tests for correlation and regression analyses. Behav. Res. Methods 2009, 41, 1149-1160. [CrossRef] [PubMed]

39. Grouzet, F.M.; Kasser, T.; Ahuvia, A.; Dols, J.M.F.; Kim, Y.; Lau, S.; Ryan, R.M.; Saunders, S.; Schmuck, P.; Sheldon, K.M. The structure of goal contents across 15 cultures. J. Pers. Soc. Psychol. 2005, 89, 800-816. [CrossRef] [PubMed]

40. Schwartz, S.H. Value priorities and behavior: Applying a theory of integrated value systems. In The Psychology of Values: The Ontario Symposium; Seligman, C., Olson, J.M., Zanna, M.P., Eds.; Lawrence Erlbaum Associates: Hillsdale, NJ, USA, 1996; Volume 8, pp. 1-24, ISBN 9781134787227.

41. Hayes, A.F. Introduction to Mediation, Moderation, and Conditional Process Analysis: A Regression-Based Approach; Guilford Press: New York, NY, USA, 2013; ISBN 978-1609182304.

42. Crompton, T.; Kasser, T. Meeting Environmental Challenges: The Role of Human Identity; WWF-UK: Godalming, UK, 2009; pp. 1-93, ISBN 9781900322645.

43. Lee, S.K.; Benavides, P.; Heo, Y.H.; Park, S.W. Narcissism increase among college students in Korea: A cross-temporal meta-analysis (1999-2014). Korean J. Psychol. Gen. 2014, 33, 609-625.

44. Park, Y.; Kim, T.S.; Park, S.W. Change in goal orientation of Korean high school athletes: A cross-temporal meta-analysis, 1999-2014. Pers. Individ. Differ. 2016, 94, 342-347. [CrossRef] 
45. Hofstede, G. National cultures in four dimensions: A research-based theory of cultural differences among nations. Int. Stud. Manag. Org. 1983, 13, 46-74. [CrossRef]

46. Rindfleisch, A.; Burroughs, J.E.; Wong, N. The safety of objects: Materialism, existential insecurity, and brand connection. J. Consum. Res. 2008, 36, 1-16. [CrossRef]

47. Shin, H.; Kim, T.; Park, Y.; Park, S.W. Materialism and mental health: A negative relation not moderated by economic status. Health Soc. Welf. Rev. 2017, 37, 368-397. [CrossRef] 